Faculty of Mathematical Sciences

\section{University of Twente}

University for Technical and Social Sciences
P.O. Box 217

7500 AE Enschede

The Netherlands

Phone: +31-53-4893400

Fax: +31-53-4893114

Email: memo@math.utwente.nl

Memorandum No. 1551

Discretization of control law

for a class of variable structure control systems

G. Golo, A.J. van Der Schaft AND

Č. Milosavluević ${ }^{1}$

November 2000

ISSN 0169-2690

${ }^{1}$ Faculty of Electrical Engineering, University of Niš, Beogradska 14, 18000 Niš, Serbia, Yugoslavia 


\title{
DISCRETIZATION OF CONTROL LAW FOR A CLASS OF VARIABLE STRUCTURE CONTROL SYSTEMS
}

\author{
G. GOLO ${ }^{1}$, A.J. VAN DER SCHAFT ${ }^{1}$, Č. MILOSAVLJEVIĆ ${ }^{2}$ \\ ${ }^{1}$ Drebbel Research Institute for Systems Engineering \\ Faculty of Mathematical Sciences, University of Twente \\ P.O. Box 217, 7500 AE Enschede \\ The Netherlands \\ ${ }^{2}$ Faculty of Electrical Engineering, University of Niš \\ Beogradska 14, 18000 Nǐs, Serbia \\ Yugoslavia
}

E-mails:g.golo@math.utwente.nl,twarjan@math.utwente.nl,milosavljevic@elfak.ni.ac.yu

A new method for the discretization of a class of continuous-time variable structure control systems, based on the linear complementarity theory, is proposed. The proposed method consists two steps. In the first step, the motion projected on the sliding manifold (the fast dynamics) is discretized by means of backward Euler time-step method. In the second step, the sampled and hold control law is determined such that the trajectories of the discrete-time closed loop system projected on the sliding manifold coincide with the trajectories of discretized fast dynamics. The discrete-time closed-loop system exhibits discrete-time sliding motion. It means that the trajectories of the discrete-time closed loop system reach the sliding manifold in a finite number of steps and stay on it after that. Also, it is proved that control law is a continuous function. Therefore, the closed loop system is chattering free. The theoretically obtained results are verified on the example of the non-holonomic integrator.

Keywords: Variable structure control systems, discretization, linear complementarity theory, discretization chatter, non-holonomic integrator.

Mathematical Subject Classification: 90C33, 93C55, 93C62, 74M05, 93C83. 


\section{Introduction}

Continuous-time variable structure control systems (CVSCS) have been extensively studied in the literature over the past forty years [5], [20], [21]. The basic features of CVSCS are invariance to parameter uncertainties and external disturbances [7], system order reduction, highly predictable behavior, etc. The design methodology for sliding mode control consists two steps [21]: selection of the sliding manifolds such that sliding motion exhibits desired properties and finding discontinuous law that enforces sliding mode in the intersections of the sliding manifolds. The techniques usually used in the second step are vector control [21], the hierarchical control [20], the reaching law method [9], etc. To implement these techniques, one often resorts to sampled data systems. However, many implementations developed for CVSCS in a sampled data environment do not achieve the performance one would expect on the basis of the continuous time theory [8]. Hence, there is a need to consider discrete-time variable structure control systems (DVSCS).

Over the last fifteen years, a few papers have been published in the area of DVSCS. Some of these papers consider how to map CVSCS into DVSCS, and their emphasis is on discretization [10], [15], [17], [18], [26], [27], [28], [25]. These approach lead to existence so-called discretization chatter that may have severe effects on the controlled system. Another line of research pursues the method of equivalent control, and based on Lyapunov stability theory, analyzes new control algorithms [1], [3], [8], [13], [19], [23].

We propose a new method for discretization of CVSCS. The proposed method consists two steps. In the first step, the motion projected on sliding manifold is discretized by means of backward Euler time-step method. In the second step, the sampled and hold control law is determined such that the trajectories of the discrete-time closed loop system projected on the sliding manifold coincide with the trajectories of discretized fast dynamics. The equations describing discretized fast dynamics are given in an implicit form. They are analyzed (existence and uniqueness of solution as well as continuity) by means of linear complementarity theory [4], [14], [16], [24]. The obtained discretized fast subsystem exhibits discrete-time sliding motion in the sense of 
definition given in [6]. The control law is a continuous function. Therefore, the closed loop system is chattering free. This method is a generalization of the results presented in [22], [12], [11].

The paper is organized in the following way: In section two, basic definitions and some results regarding linear complementarity theory are recalled. The class of the system considered in this paper is described in the section three. The discretization of the motion projected on the sliding manifold as well as the determining of control law is presented in the section four. Some features of obtained control law are analyzed in the section five. The theoretically obtained results are verified on the example of non-holonomic integrator and some simulation results are presented in the section six.

\section{Mathematical preliminaries}

This section starts with recalling the formulation of the linear complementarity problem [4].

\section{Linear complementarity problem $(\mathrm{LCP}(\boldsymbol{q}, \boldsymbol{M}))$}

Given a matrix $\boldsymbol{M} \in \mathcal{R}^{m \times m}$ and a vector $\boldsymbol{q} \in \mathcal{R}^{m}$, find the vectors $\boldsymbol{w}, \boldsymbol{r} \in \mathcal{R}^{m}$ such that

$$
\begin{aligned}
& \boldsymbol{w}=\boldsymbol{q}+\boldsymbol{M} \boldsymbol{r} \\
& \boldsymbol{w} \geq 0 \\
& \boldsymbol{r} \geq 0 \\
& \boldsymbol{r}^{\mathrm{T}} \boldsymbol{w}=0 .
\end{aligned}
$$

or show that no such vector exists. In this definition inequalities should be consider to hold component wise, e.g. $\boldsymbol{w} \geq 0$ means $w_{i} \geq 0, i=\overline{1, m}$.

We introduce some further definitions and recall some results concerning LCP .

Definition 1: Let $\boldsymbol{M} \in \Re^{m \times m}$. For index sets $\mathcal{I} \subseteq\{1,2, \ldots, m\}, \mathcal{J} \subseteq\{1,2, \ldots, m\}$ the sub matrix $\boldsymbol{M}_{\mathcal{I J}}$ of $\boldsymbol{M}$ is the matrix whose entries lie in the rows of $\boldsymbol{M}$ indexed by $\mathcal{I}$ and the columns of $\boldsymbol{M}$ indexed by $\mathcal{J}$.

Definition 2: Given a matrix $M \in \Re^{m \times m}$ and two nonempty subsets $\mathcal{I}$ and $\mathcal{J}$ of $\{1,2, \ldots, m\}$ with the same cardinality, the $(\mathcal{I}, \mathcal{J})$-minor of $\boldsymbol{M}$ is the determinant of the square sub matrix $\boldsymbol{M}_{\mathcal{I J}}$. The principal minors are those with $\mathcal{I}=\mathcal{J}$. 
Definition 3: A matrix $\boldsymbol{M} \in \Re^{n \times n}$ is said to be a $\boldsymbol{P}$-matrix if all its principal minors are positive. $\boldsymbol{M}$ is said to be a $\boldsymbol{P}_{o}$-matrix if all its principal minors are nonnegative.

Theorem 1: ([14]) Suppose that a matrix $\boldsymbol{N} \in \Re^{m \times m}$ is a $\boldsymbol{P}$-matrix. Then $\operatorname{LCP}(\boldsymbol{q}, \boldsymbol{M})$ where, $\boldsymbol{q} \in \Re^{2 m}$ and $\boldsymbol{M}=\left[\begin{array}{cc}\boldsymbol{N}^{-1} & -\boldsymbol{N}^{-1} \\ -\boldsymbol{N}^{-1} & \boldsymbol{N}^{-1}\end{array}\right]$

a) is solvable for every $\boldsymbol{q} \in \Re^{m}$,

b) has the unique solution that satisfies the conditions $\boldsymbol{r}_{1}^{\mathrm{T}} \boldsymbol{r}_{2}=0$, for every $\boldsymbol{q} \in \Re^{m}$.

Here, $\boldsymbol{r}_{1}$ represents the first $m$ components of the vector $\boldsymbol{r}$, and $\boldsymbol{r}_{2}$ represents the other $m$ components of the vector $\boldsymbol{r}$. Let us consider a multi-valued function defined by

$$
h=\operatorname{Sgn}(s) \in\left\{\begin{array}{c}
\{1\}, s>0 \\
{[-1,1], s=0 .} \\
\{-1\}, s>0
\end{array}\right.
$$

Let the variables $h^{+}, h^{-}, s^{+}, s^{-}$be defined as [14]

$$
\begin{aligned}
h^{+} & =1-h, \\
h^{-} & =1+h, \\
s^{+} & =\frac{1}{2}(|s|+s), \\
s^{-} & =\frac{1}{2}(|s|-s) .
\end{aligned}
$$

The relation (2) implies that $h^{+}+h^{-}=2$ and $s^{+} s^{-}=0$. If $h$ and $s$ are connected by the relation (1) then it can be easily shown that $h^{+}, h^{-}, s^{+}, s^{-}$are nonnegative numbers satisfying the following equality

$$
\left[\begin{array}{ll}
s^{+} & s^{-}
\end{array}\right]\left[\begin{array}{l}
h^{+} \\
h^{-}
\end{array}\right]=0 \text {. }
$$

From the relation (2), the expressions for $h, s$ can be derived as

$$
\begin{aligned}
& h=\frac{1}{2}\left(h^{-}-h^{+}\right), \\
& s=s^{+}-s^{-} .
\end{aligned}
$$

It is possible to prove that if the relation (3) is satisfied, $h^{+}, h^{-}, s^{+}, s^{-}$are nonnegative numbers such that $h^{+}+h^{-}=2$ and $h^{+} h^{-}=0$ then (4) implies that $h=\operatorname{Sgn}(s)$. 


\section{Continuous-time variable structure control systems}

A class of systems considered in this paper is described by

$$
\dot{\boldsymbol{x}}=\boldsymbol{f}(\boldsymbol{x})+\boldsymbol{G}(\boldsymbol{x}) \boldsymbol{u} .
$$

The state vector $\boldsymbol{x}$ belongs to an $n$-dimensional differentiable manifold $\mathcal{X}$. The vector $\boldsymbol{f}$ and the matrix $\boldsymbol{G}$ are smooth mappings on $\mathcal{X} . \boldsymbol{u}$ is control signal and $\boldsymbol{u} \in \Re^{m}$. The control objective is to steer the trajectory of (1) to $n-m$ dimensional manifold $\mathcal{S}$ defined by

$$
\mathcal{S}=\left\{\boldsymbol{x} \in \mathcal{X}: s_{i}(\boldsymbol{x})=0, i=\overline{1, m}\right\} .
$$

in finite time for every initial condition $\boldsymbol{x}_{\mathrm{o}} \in \mathcal{X}$. Here, $s_{i}(\boldsymbol{x}), i=\overline{1, m}$ is a smooth scalar function defined on $\mathcal{X}$. It is assumed that

$$
\begin{aligned}
& \operatorname{det}\left(\frac{\partial \boldsymbol{s}(\boldsymbol{x})}{\partial \boldsymbol{x}} \boldsymbol{G}(\boldsymbol{x})\right) \neq 0, \forall \boldsymbol{x} \in \mathcal{X}, \\
& \boldsymbol{s}^{\mathrm{T}}(\boldsymbol{x})=\left[\begin{array}{lll}
s_{1}(\boldsymbol{x}) & \cdots & s_{m}(\boldsymbol{x})
\end{array}\right] .
\end{aligned}
$$

The condition (7) (usual condition for VSCS) ensures that the system (5) together with the output $\boldsymbol{y}=\boldsymbol{s}(\boldsymbol{x})$ is strongly input-output decoupled. Also this condition guarantees that the motion of the system (5) along the sliding manifold is uniquely defined. Suppose that control law that achieves above mentioned control goal is given by

$$
\boldsymbol{u}(\boldsymbol{x})=\boldsymbol{u}^{l}(\boldsymbol{x})-\Delta \boldsymbol{U}(\boldsymbol{x}) \operatorname{Sgn}(\boldsymbol{s}) .
$$

Here, $\operatorname{Sgn}(\boldsymbol{s})$ stands for $\left[\operatorname{Sgn}\left(s_{1}\right) \cdots \operatorname{Sgn}\left(s_{m}\right)\right]^{\mathrm{T}}$ and $\operatorname{det}(\Delta \boldsymbol{U}(\boldsymbol{x})) \neq 0, \forall \boldsymbol{x} \in \mathcal{X}$. The motion projection on the subspace $\mathcal{S}$ is given by [20]

$$
\begin{aligned}
& \dot{\boldsymbol{s}}=\boldsymbol{d}(\boldsymbol{x})-\boldsymbol{D}(\boldsymbol{x}) \operatorname{Sgn}(\boldsymbol{s}), \\
& \boldsymbol{d}(\boldsymbol{x})=\frac{\partial \boldsymbol{s}(\boldsymbol{x})}{\partial \boldsymbol{x}} \boldsymbol{f}(\boldsymbol{x})+\frac{\partial \boldsymbol{s}(\boldsymbol{x})}{\partial \boldsymbol{x}} \boldsymbol{G}(\boldsymbol{x}) \boldsymbol{u}^{l}(\boldsymbol{x}), \\
& \boldsymbol{D}(\boldsymbol{x})=\frac{\partial \boldsymbol{s}(\boldsymbol{x})}{\partial \boldsymbol{x}} \boldsymbol{G}(\boldsymbol{x}) \Delta \boldsymbol{U}(\boldsymbol{x}) .
\end{aligned}
$$

From (9) one may conclude that if the control law is known then the equation describing the motion projection on $\mathcal{S}$ is uniquely defined. Since the relation (7) is satisfied, then the opposite is true, i.e. the equation (9) uniquely determines the control law (8). Finally, it is assumed the functions $\boldsymbol{u}_{l}(\boldsymbol{x}), \Delta \boldsymbol{U}(\boldsymbol{x})$ are chosen such that $\boldsymbol{d}(\boldsymbol{x}), \boldsymbol{D}(\boldsymbol{x})$ satisfy the following conditions

$$
\left\|\boldsymbol{D}^{-1}(\boldsymbol{x}) \boldsymbol{d}(\boldsymbol{x})\right\|_{\infty} \leq 1, \forall \boldsymbol{x} \in \mathcal{S},
$$




$$
\lambda_{\min }\left(\frac{1}{2}\left(\boldsymbol{D}(\boldsymbol{x})+\boldsymbol{D}^{\mathrm{T}}(\boldsymbol{x})\right)\right)-\|\boldsymbol{d}(\boldsymbol{x})\|_{1} \geq \lambda_{\circ}>0, \forall \boldsymbol{x} \in \mathcal{X} .
$$

First condition ensures that sliding mode exists on the manifold $\mathcal{S}$. The later one guarantees that the trajectories of (5) reach the sliding manifold $\mathcal{S}$ in finite time (see e.g. [20]).

\section{Discretization}

The equation (9) is approximated by a difference equation (backward Euler time stepping method) as

$$
\boldsymbol{s}_{k+1}=\boldsymbol{s}_{k}+\operatorname{Td}\left(\boldsymbol{x}_{k}\right)-T \boldsymbol{D}\left(\boldsymbol{x}_{k}\right) \operatorname{Sgn}\left(\boldsymbol{s}_{k+1}\right) .
$$

Here, $\boldsymbol{s}_{k}, \boldsymbol{x}_{k}$ stands for the value of $\boldsymbol{s}, \boldsymbol{x}$ in the moment $k T$ and the positive number $T$ stands for the sampling period. Note that this discretization scheme for the single input systems is proposed in [22]. By observing the equation (12), one may conclude that it represents an implicit equation in respect to $s_{k+1}$. Therefore the following questions have to be answered:

- Does the equation (12) is solvable for $\boldsymbol{s}_{k+1}$ ?

- What can be said about uniqueness of the solution?

- How can one practically find the solution of (12)?

To answer the first two questions, the equation (12) is transformed into the following form

$$
\operatorname{Sgn}\left(s_{k+1}\right)=\boldsymbol{D}^{-1}\left(\boldsymbol{x}_{k}\right)\left(\frac{\boldsymbol{s}_{k}}{T}+\boldsymbol{d}\left(\boldsymbol{x}_{k}\right)\right)-T^{-1} \boldsymbol{D}^{-1}\left(\boldsymbol{x}_{k}\right) \boldsymbol{s}_{k+1} .
$$

The matrix $\boldsymbol{D}(\boldsymbol{x})$ is invertible matrix $\forall \boldsymbol{x} \in \mathcal{X}$, since the relation (7) is satisfied and the matrix $\Delta \boldsymbol{U}(\boldsymbol{x})$ is regular for every $\boldsymbol{x} \in \mathcal{X}$. Applying the equation (2) to (13) one may find the following

$$
\begin{gathered}
\boldsymbol{w}=\boldsymbol{q}+\boldsymbol{M r}, \\
\boldsymbol{w}=\left[\begin{array}{l}
\boldsymbol{w}_{1} \\
\boldsymbol{w}_{2}
\end{array}\right]=\left[\begin{array}{l}
\boldsymbol{h}_{k}^{+} \\
\boldsymbol{h}_{k}^{-}
\end{array}\right]=\left[\begin{array}{l}
1_{m}-\operatorname{Sgn}\left(\boldsymbol{s}_{k+1}\right) \\
1_{m}+\operatorname{Sgn}\left(\boldsymbol{s}_{k+1}\right)
\end{array}\right], \boldsymbol{r}=\left[\begin{array}{l}
\boldsymbol{r}_{1} \\
\boldsymbol{r}_{2}
\end{array}\right]=\left[\begin{array}{l}
\boldsymbol{s}_{k+1}^{+} \\
\boldsymbol{s}_{k+1}^{-}
\end{array}\right], \\
\boldsymbol{q}=\left[\begin{array}{c}
1_{m}-\boldsymbol{D}^{-1}\left(\boldsymbol{x}_{k}\right)\left(\frac{\boldsymbol{s}_{k}}{T}+\boldsymbol{d}\left(\boldsymbol{x}_{k}\right)\right) \\
1_{m}+\boldsymbol{D}^{-1}\left(\boldsymbol{x}_{k}\right)\left(\frac{\boldsymbol{s}_{k}}{T}+\boldsymbol{d}\left(\boldsymbol{x}_{k}\right)\right)
\end{array}\right], \boldsymbol{M}=\left[\begin{array}{cc}
\boldsymbol{N}^{-1} & -\boldsymbol{N}^{-1} \\
-\boldsymbol{N}^{-1} & \boldsymbol{N}^{-1}
\end{array}\right] \\
\boldsymbol{N}=T \boldsymbol{D}\left(\boldsymbol{x}_{k}\right) .
\end{gathered}
$$


Here, $1_{m}$ denotes $m$-dimensional vector which entries are one. If $\boldsymbol{h}_{k}=\operatorname{Sgn}\left(\boldsymbol{s}_{k+1}\right)$ and transformation (2) is applied then (see section 2) $\boldsymbol{w}^{\mathrm{T}} \boldsymbol{r}=0, \boldsymbol{w}, \boldsymbol{r} \geq 0$, $\boldsymbol{r}_{1}^{\mathrm{T}} \boldsymbol{r}_{2}=0$ and $\boldsymbol{w}_{1}+\boldsymbol{w}_{2}=2 \cdot 1_{m}$. Therefore the problem of solving (12) for $s_{k+1}$, is reduced on the solving $\operatorname{LCP}(\boldsymbol{q}, \boldsymbol{M})$ where $\boldsymbol{q}$ and $\boldsymbol{M}$ are given by (14) and vector $\boldsymbol{r}$ satisfies the relation $\boldsymbol{r}_{1}^{\mathrm{T}} \boldsymbol{r}_{2}=0$ (Notice that if there is a solution of $\operatorname{LCP}(\boldsymbol{q}, \boldsymbol{M})$ then it also satisfies the condition $\left.\boldsymbol{w}_{1}+\boldsymbol{w}=2 \cdot 1\right)$. Since the matrix $\boldsymbol{D}(\boldsymbol{x})$ satisfies the condition (10), it is a $\boldsymbol{P}$-matrix $\forall \boldsymbol{x} \in \mathcal{X}$. It means that the matrix $\boldsymbol{N}$ is a $\boldsymbol{P}$-matrix and $\operatorname{LCP}(\boldsymbol{q}, \boldsymbol{M})$ is solvable (Theorem 1 part a) and solution that satisfies the condition $\boldsymbol{r}_{1}^{\mathrm{T}} \boldsymbol{r}_{2}=0$ is unique (Theorem 1 part b). Furthermore, by using the relation (4) we can determine the value of $\boldsymbol{s}_{k+1}=\boldsymbol{r}_{1}-\boldsymbol{r}_{2}$ and $\operatorname{Sgn}\left(\boldsymbol{s}_{k+1}\right)=\frac{1}{2}\left(\boldsymbol{w}_{2}-\boldsymbol{w}_{1}\right)$. Therefore, the equation (12) can be transformed into an explicit form as

$$
\boldsymbol{s}_{k+1}=\Phi\left(\boldsymbol{s}_{k}, \boldsymbol{x}_{k}, T\right)=\boldsymbol{s}_{k}+\operatorname{Td}\left(\boldsymbol{x}_{k}\right)-T \boldsymbol{D}\left(\boldsymbol{x}_{k}\right) \varphi\left(\boldsymbol{s}_{k}, \boldsymbol{x}_{k}, T\right) .
$$

$\operatorname{LCP}(\boldsymbol{q}, \boldsymbol{M})$ can be practically solved by using the pivoting method or the iterative techniques. However, for the scalar case, $m=1$, it is possible to find the solution in a closed form. If $m=1$ the equation (12) can be rewritten as

$$
s_{k+1}=s_{k}+T d\left(\boldsymbol{x}_{k}\right)-T D\left(\boldsymbol{x}_{k}\right) \operatorname{Sgn}\left(s_{k+1}\right) \text {. }
$$

Here, $d(x)$ and $D(x)$ are scalars. $D(x)$ is a $\boldsymbol{P}$-matrix which means that $D(\boldsymbol{x})$ is positive for every $\boldsymbol{x} \in \mathcal{X}$. Let $s_{k+1}>0$. Then $\operatorname{Sgn}\left(s_{k+1}\right)=1$ and $s_{k+1}$ can be determined from (16) as $s_{k+1}=s_{k}+T d\left(\boldsymbol{x}_{k}\right)-T D\left(\boldsymbol{x}_{k}\right)$. Since $s_{k+1}>0$ then $s_{k}+T d\left(\boldsymbol{x}_{k}\right)>T D\left(\boldsymbol{x}_{k}\right)$. Similarly, if $s_{k}+T d\left(\boldsymbol{x}_{k}\right)<-T D\left(\boldsymbol{x}_{k}\right)$ then $s_{k+1}<0$ and $s_{k+1}=s_{k}+T d\left(\boldsymbol{x}_{k}\right)+T D\left(\boldsymbol{x}_{k}\right)$. If $s_{k+1}=0$ then $\operatorname{Sgn}\left(s_{k+1}\right)=\frac{s_{k}+T d\left(\boldsymbol{x}_{k}\right)}{T D\left(\boldsymbol{x}_{k}\right)}$. Since $\operatorname{Sgn}\left(s_{k+1}\right) \in[-1,1]$ then $\left|\frac{s_{k}+T d\left(\boldsymbol{x}_{k}\right)}{T D\left(\boldsymbol{x}_{k}\right)}\right| \leq 1$. The previous considered cases can be rewritten in a condensed form as

$$
s_{k+1}=s_{k}+T d\left(\boldsymbol{x}_{k}\right)-T D\left(\boldsymbol{x}_{k}\right) \min \left(\left|\frac{s_{k}+T d\left(\boldsymbol{x}_{k}\right)}{T D\left(\boldsymbol{x}_{k}\right)}\right|, 1\right) \operatorname{sgn}\left(s_{k}+T d\left(\boldsymbol{x}_{k}\right)\right) .
$$

The same solution can be also found from the equation (14) as shown in [16]. The mapping $\varphi(\boldsymbol{x}, s, T)$ for some specific forms of $d(\boldsymbol{x})$ and $D(\boldsymbol{x})$ is given in 
Table 1. The solution for the scalar case can be easily applied on the vector case if the matrix $\boldsymbol{D}(\boldsymbol{x})$ is either upper or lower triangular.

Table 1. The form of $\varphi$ for some specific forms of $d$ and $D$

\begin{tabular}{|c|c|c|c|c|c|c|c|}
\hline & $d(\boldsymbol{x})$ & $D(\boldsymbol{x})$ & \multicolumn{5}{|c|}{$\varphi(s, \boldsymbol{x}, T)$} \\
\hline 1 & 0 & $\sigma, \sigma>0$ & & $\min$ & $\left.\frac{|s|}{\sigma T}, 1\right) \mathrm{s}$ & $\operatorname{gn}(s)$ & \\
\hline 2 & $-\sigma_{1} s, \sigma_{1}>0$ & $\sigma_{2}, \sigma_{2}>0$ & & $\begin{array}{l}\left(\frac{|s|(1}{c}\right. \\
1-\sigma\end{array}$ & $\begin{array}{l}\left.-\sigma_{1} T\right) \\
{ }_{2} T \\
T<1\end{array}$ & sgn & $(s)$, \\
\hline 3 & 0 & $\begin{array}{l}\sigma+\sum_{i=1}^{n} \sigma_{i}\left|x_{i}\right| \\
\sigma>0, \sigma_{i}>0, i=\overline{1, n}\end{array}$ & $\min$ & $\sigma T+$ & $\frac{|s|}{T \sum_{i=1}^{n} \sigma_{i} \mid x}$ & $-c_{i} \mid, 1$ & $\operatorname{sgn}(s)$ \\
\hline
\end{tabular}

Now, it will be explained how previous obtained results can be used in determining the control signal $\boldsymbol{u}$. Let the sampled and hold control signal

$$
\boldsymbol{u}(t)=\boldsymbol{u}_{k}=\boldsymbol{u}(k T), t \in[k T,(k+1) T),
$$

is applied on the system described by (5). The exact discrete-time model in the time instants $k T$ is given by

$$
\begin{aligned}
& \boldsymbol{x}_{k+1}=\boldsymbol{F}\left(\boldsymbol{x}_{k}, \boldsymbol{u}_{k}, T\right) . \\
& \boldsymbol{x}_{0}=\boldsymbol{x}_{\mathrm{o}}
\end{aligned}
$$

Here, $\boldsymbol{F}(\boldsymbol{x}, \boldsymbol{u}, T)$ is a smooth function and it is clear that $\lim _{T \rightarrow 0} \frac{\boldsymbol{F}(\boldsymbol{x}, \boldsymbol{u}, T)-\boldsymbol{x}}{T}=\boldsymbol{f}(\boldsymbol{x})+\boldsymbol{G}(\boldsymbol{x}) \boldsymbol{u}$. From (19) $\boldsymbol{s}_{k+1}$ is given by

$$
\boldsymbol{s}_{k+1}=\boldsymbol{s}\left(\boldsymbol{x}_{k+1}\right)=\boldsymbol{s}\left(\boldsymbol{F}\left(\boldsymbol{x}_{k}, \boldsymbol{u}_{k}, T\right)\right) .
$$

Since the condition (7) is satisfied then for enough small $T$, the equation (20) can be solved for $\boldsymbol{u}_{k}$ as

$$
\boldsymbol{u}_{k}=\Psi\left(\boldsymbol{x}_{k}, \boldsymbol{s}_{k}, \boldsymbol{s}_{k+1}, T\right) .
$$

Here, $\Psi$ is a smooth mapping. Therefore, the value of control signal in the step $k$ can be find by using the equations (15) and (21), as

$$
\boldsymbol{u}_{k}=\Psi\left(\boldsymbol{x}_{k}, \boldsymbol{s}_{k}, \Phi\left(\boldsymbol{x}_{k}, \boldsymbol{s}_{k}, T\right), T\right)=\Theta\left(\boldsymbol{x}_{k}, \boldsymbol{s}_{k}, T\right) .
$$




\section{Stability in finite time and continuity}

In this section some asymptotically properties of the closed-loop system described by (19) (21) and (15) are analyzed. Also the nature of the mapping $\Phi$ is investigated. The obtained results are summarized in the next two theorems.

Theorem 2: Let consider discrete-time system described by (19) where the control law is described by the relations (21), (15) and the conditions (10), (11) are satisfied. Then for every initial condition $\boldsymbol{x}_{\mathrm{o}} \in \mathcal{X}$ the point $\boldsymbol{x}$ reaches the manifold $\mathcal{S}$ in a finite number of steps and stays on it after that.

Proof The motion projection of the closed-loop system (19) (21) and (15) on the manifold $\mathcal{S}$ is given by the equation (15). Firstly, let consider the case when $\boldsymbol{x}_{k} \in \mathcal{S}$. Then $\boldsymbol{s}_{k}=0$ and $\boldsymbol{s}_{k+1}$ can be find as the solution of the equation

$$
\boldsymbol{s}_{k+1}=\operatorname{Td}\left(\boldsymbol{x}_{k}\right)-\boldsymbol{D}\left(\boldsymbol{x}_{k}\right) \operatorname{Sgn}\left(\boldsymbol{s}_{k+1}\right) .
$$

If $\boldsymbol{s}_{k+1}=0$ then the last equation implies that $\operatorname{Sgn}\left(s_{k+1}\right)=\boldsymbol{D}^{-1}\left(\boldsymbol{x}_{k}\right) \boldsymbol{d}\left(\boldsymbol{x}_{k}\right)$. Since the condition (10) is satisfied then every component of the vector $\boldsymbol{D}^{-1}\left(\boldsymbol{x}_{k}\right) \boldsymbol{d}\left(\boldsymbol{x}_{k}\right)$ belongs to the segment $[-1,1]$. Therefore, $s_{k+1}=0$ is indeed a solution of $(23)$. This solution is also unique since the matrix $\boldsymbol{D}\left(\boldsymbol{x}_{k}\right)$ is a $\boldsymbol{P}$-matrix. Let $\boldsymbol{s}_{k} \notin \mathcal{S}$. $V(\boldsymbol{s})=\|\boldsymbol{s}\|_{1}=\sum_{i=1}^{m}\left|s_{i}\right|$ is consider as a candidate for the Lyapunov function. The first difference $\Delta V_{k}=V_{k+1}-V_{k}=V\left(s_{k+1}\right)-V\left(s_{k}\right)$ is given by

$$
\begin{aligned}
& \Delta V_{k}=\left(\operatorname{Sgn}\left(\boldsymbol{s}_{k+1}\right)\right)^{\mathrm{T}} \boldsymbol{s}_{k+1}-\left\|\boldsymbol{s}_{k}\right\|_{1}= \\
& \left(\operatorname{Sgn}\left(\boldsymbol{s}_{k+1}\right)\right)^{\mathrm{T}} \boldsymbol{d}\left(\boldsymbol{x}_{k}\right)-\left(\operatorname{Sgn}\left(\boldsymbol{s}_{k+1}\right)\right)^{\mathrm{T}} \boldsymbol{D}\left(\boldsymbol{x}_{k}\right) \operatorname{Sgn}\left(\boldsymbol{s}_{k+1}\right) \\
& -\sum_{i=1}^{m}\left|s_{k, i}\right| \underbrace{\left(1-\operatorname{Sgn}\left(s_{k, i}\right) \operatorname{Sgn}\left(s_{k+1, i}\right)\right)}_{\in[0,2]} .
\end{aligned}
$$

Suppose now that $s_{k+1} \notin \mathcal{S}$ then there is at least one component of the vector $s_{k+1}$ different from zero. It implies that at last one component of the vector $\operatorname{Sgn}\left(s_{k+1}\right)$ is \pm 1 . Hence, by using the condition (11) the second raw of (24) is smaller then $-T \lambda_{\circ}$. It means that $\Delta V_{k} \leq-\lambda_{o} T$ since the third row of (24) is always non-positive. This implies that there is a number $K_{\mathrm{o}}$ such that $s_{K_{\mathrm{o}}} \in \mathcal{S}$ and consequently $s_{k} \in \mathcal{S}, k \geq K_{\mathrm{o}}+1$. 
Next theorem analyzes the continuity of the mapping $\Phi$. It is proved that $\Phi$ is a continuous mapping in respect to $\boldsymbol{x}, \boldsymbol{s}, T$. Continuity of the mapping $\Phi$ implies also the continuity of the mapping $\Theta$, therefore, the control law is continuous function in respect to $\boldsymbol{x}, \boldsymbol{s}, T$. Hence the control law does not generate discretization chatter.

Theorem 3 The mapping $\Phi$ is continuous function in respect to $\boldsymbol{x}, s, T$.

Proof The sketch of the proof is given only.

- If we prove that the solution of $\operatorname{LCP}(\boldsymbol{q}, \boldsymbol{M})$ is continuous function in respect to $\boldsymbol{q}$ and $\boldsymbol{M}$ then the mapping $\Phi$ is continuous function in respect to $\boldsymbol{x}, \boldsymbol{s}, T$ since $\boldsymbol{q}$ and $\boldsymbol{M}$ are continuous functions in respect to $\boldsymbol{x}, \boldsymbol{s}, T$.

- Suppose that the solution of $\operatorname{LCP}(\boldsymbol{q}, \boldsymbol{M})$ is not continuous function in the point $\left(\boldsymbol{q}^{*}, \boldsymbol{M}^{*}\right)$. Then exists a sequence $\left\{\left(\boldsymbol{q}^{i}, \boldsymbol{M}^{i}\right)\right\}_{i=0}^{\infty}$ and a positive number $\varepsilon$ such that $\left\|\boldsymbol{r}^{*}-\boldsymbol{r}^{i}\right\| \geq \varepsilon, \forall i \in \overline{1, \infty}$. Here, $\boldsymbol{r}^{*}, \boldsymbol{r}^{i}$ stand for the solution of $\operatorname{LCP}\left(\boldsymbol{q}^{*}, \boldsymbol{M}^{*}\right)$ and $\operatorname{LCP}\left(\boldsymbol{q}^{i}, \boldsymbol{M}^{i}\right)$, respectively.

- It can be proved that $\operatorname{LCP}(0, \boldsymbol{M})$ has only one trivial solution $\boldsymbol{r}=0$ that satisfies the condition $\boldsymbol{r}_{1}^{\mathrm{T}} \boldsymbol{r}_{2}=0$.

- The fact that $\operatorname{LCP}(0, \boldsymbol{M})$ has only one trivial solution can help us to prove that the sequence $\left\{\boldsymbol{r}^{i}\right\}_{i=0}^{\infty}$ is bounded sequence. Therefore, there is a subsequence $\quad\left\{\left(\boldsymbol{q}^{i_{j}}, \boldsymbol{M}^{i_{j}}\right)\right\}_{j=0}^{\infty} \subset\left\{\left(\boldsymbol{q}^{i}, \boldsymbol{M}^{i}\right)\right\}_{i=0}^{\infty} \quad$ such that solution of $\operatorname{LCP}\left(\boldsymbol{q}^{i_{j}}, \boldsymbol{M}^{i_{j}}\right), \quad \boldsymbol{r}^{i_{j}}$, tends to the point $\overline{\boldsymbol{r}}$. However, since $\lim _{j \rightarrow \infty}\left(\boldsymbol{q}^{i_{j}}, \boldsymbol{M}^{i_{j}}\right)=\left(\boldsymbol{q}^{*}, \boldsymbol{M}^{*}\right)$ then $\operatorname{LCP}\left(\boldsymbol{q}^{*}, \boldsymbol{M}^{*}\right)$ has two different solution, $\boldsymbol{r}^{*}, \overline{\boldsymbol{r}}$. This is the contradiction, therefore the solution of $\operatorname{LCP}(\boldsymbol{q}, \boldsymbol{M})$ is continuous function in respect to $\boldsymbol{q}$ and $\boldsymbol{M}$.

If the control law described by (18), (21), (15) is applied on the system (5) then there is a number $K_{\mathrm{o}}$ such that $s(k T)=0, k \geq K_{\mathrm{o}}$. However, it does not mean that $s(t)=0, t \geq K_{\mathrm{o}} T$. If the motion of the closed-loop system (5), (8) on the manifold $\mathcal{S}$ is stable then it is possible to show that there is a constant $\eta$ such that $\|s(t)\|_{2} \leq \eta T, t \geq K_{\mathrm{o}} T$. 


\section{Example}

The following example is taken from [2, 24]. It represents the model of nonholonomic integrator. To indicate the physical significance of this system it can be shown that any non-holonomic mechanical system with three states and two inputs can be converted in this form. This system is the prototype of a nonlinear system, which is controllable, but nevertheless cannot be stabilized using the differentiable state feedback (static or dynamic) [24].

The system is described by

$$
\begin{aligned}
& \dot{x}=u, \\
& \dot{y}=v, \\
& \dot{z}=x v-y u .
\end{aligned}
$$

In [2] is shown that control law

$$
\begin{aligned}
& u=-\alpha x+\beta y \operatorname{sgn}(z), \\
& v=-\alpha y-\beta x \operatorname{sgn}(z), \\
& \alpha, \beta>0
\end{aligned}
$$

stabilizes system (25) for every initial condition that satisfies the following condition

$$
\frac{\beta}{2 \alpha}\left(x_{\mathrm{o}}^{2}+y_{\mathrm{o}}^{2}\right)<\left|z_{\mathrm{o}}\right| \text {. }
$$

The sliding manifold is plane $z=0$. The motion projection on the sliding manifold is given by

$$
\dot{z}=-\beta \underbrace{\left(x^{2}+y^{2}\right)}_{2 V} \operatorname{sgn}(z)=-2 \beta V \operatorname{sgn}(z) .
$$

The discretized fast dynamics is given by

$$
z_{k+1}=z_{k}-2 \beta T V_{k} \operatorname{Sgn}\left(z_{k+1}\right) .
$$

By using results obtained in the section 4 the last equation is equivalent to

$$
z_{k+1}=z_{k}-\min \left(\left|z_{k}\right|, 2 \beta T V_{k}\right) \operatorname{sgn}\left(z_{k}\right) .
$$

If we apply sampled-hold control on the system (25), the following exact discrete-time model is obtained

$$
\begin{aligned}
& x_{k+1}=x_{k}+T u_{k}, \\
& y_{k+1}=y_{k}+T v_{k}, \\
& z_{k+1}=z_{k}+T\left(x_{k} v_{k}-y_{k} u_{k}\right), \\
& {\left[\begin{array}{lll}
x_{0} & y_{0} & z_{0}
\end{array}\right]^{T}=\left[\begin{array}{lll}
x_{\mathrm{o}} & y_{\mathrm{o}} & z_{\mathrm{o}}
\end{array}\right]^{\mathrm{T}}}
\end{aligned}
$$


From (29) and (30) one may conclude that $u_{k}, v_{k}$ have to be chosen so that the following relation is satisfied

$$
y_{k} u_{k}-x_{k} v_{k}=\min \left(\left|z_{k}\right|, 2 \beta T V_{k}\right) \operatorname{sgn}\left(z_{k}\right) .
$$

One possible solution is given by

$$
\begin{aligned}
& u_{k}=-\alpha x_{k}+\beta y_{k} \min \left(\frac{\left|z_{k}\right|}{2 \beta T V_{k}}, 1\right) \operatorname{sgn}\left(z_{k}\right) \\
& v_{k}=-\alpha y_{k}-\beta x_{k} \min \left(\frac{\left|z_{k}\right|}{2 \beta T V_{k}}, 1\right) \operatorname{sgn}\left(z_{k}\right)
\end{aligned} .
$$

From (31), (30) it is possible to obtained that

$$
V_{k+1}=\frac{1}{2}\left(x_{k+1}^{2}+y_{k+1}^{2}\right)=\left((1-\alpha T)^{2}+\left(\beta T \min \left(\frac{\left|z_{k}\right|}{2 \beta T V_{k}}, 1\right)\right)^{2}\right) V_{k} .
$$

The state $z_{k}$ becomes zero in a finite number of steps if there is a number $K_{\text {o }}$ such that $\left|z_{K_{\mathrm{o}}}\right| \leq 2 \beta T V_{K_{\mathrm{o}}}$. Using the equations (29), (32) the last conditions can be rewritten as

$$
\begin{aligned}
& \left|z_{\mathrm{o}}\right| \leq 2 \beta T V_{0}\left(1+a+\ldots+a^{K_{\mathrm{o}}-1}+2 a^{K_{\mathrm{o}}}\right), \\
& a=(1-\alpha T)^{2}+\beta^{2} T^{2} .
\end{aligned}
$$

If $a<1 \Leftrightarrow T<\frac{2 \alpha}{\alpha^{2}+\beta^{2}}$ then sufficient and necessary condition guaranteeing $z_{k}$ becomes 0 in the finite number of steps is given by

$$
\left|z_{\mathrm{o}}\right| \leq 2 \beta T V_{0} \lim _{K_{\mathrm{o}} \rightarrow \infty}\left(1+a+\ldots+a^{K_{\mathrm{o}}-1}+2 a^{K_{\mathrm{o}}}\right)=\frac{2 \beta T}{1-a} V_{0} .
$$

Obviously, if $T \rightarrow 0$ then the condition (34) transforms into (27). If $a \geq 1 \Leftrightarrow T \geq \frac{2 \alpha}{\alpha^{2}+\beta^{2}}$ then for every $x_{\mathrm{o}}^{2}+y_{\mathrm{o}}^{2} \neq 0$ there exists a number $K$ such that the conditions (33) is satisfied. Suppose that the state $z_{k}$ becomes zero in finite number of steps, i.e. $z_{k}=0, k>K_{\mathrm{o}}$. Then the relation (32) becomes

$$
V_{k+1}=(1-\alpha T)^{2} V_{k}, k \geq K_{\mathrm{o}}+1 .
$$

If the parameters $\alpha, T$ are chosen such that

$$
0<\alpha T<2
$$

then $V_{k}$ exponentially converges to zero which means also that the states $x_{k}, y_{k}$ exponentially converge to zero. One may see from the equation (30) that states of the system described by (25) are linear function on the segment $[k T,(k+1) T]$ if the sampled and hold control is applied. Since, we have proved that $z_{k}$ 
converge to zero in finite time and $x_{k}, y_{k}$ exponentially tends to zero then $z(t)$ becomes zero in finite time and $x(t), y(t)$ asymptotically tend to zero. In this way, by choosing the appropriate values of the parameters $\alpha, \beta, T$, the system (25) can be stabilize for larger area of initial conditions then one obtained by applying the control law (26).

To check previous obtained results numerical simulation is carried out. The parameters $\alpha, \beta$ are 1,2, respectively. The sampling period $T$ has to belong to the interval $\left(0,2 \alpha^{-1}\right)=(0,2)$ (the equation (35)). The initial condition is $\left[\begin{array}{lll}2 & 2 & 3\end{array}\right]^{\mathrm{T}}$. For this initial condition the system (25) is stabilized for every $T \in(0,2)$. The numerical simulation is performed for three values of the sampling period: $T \in\{0.005,0.05,0.5\}$. The results of the numerical simulation are given in Figure 1 to Figure 4.

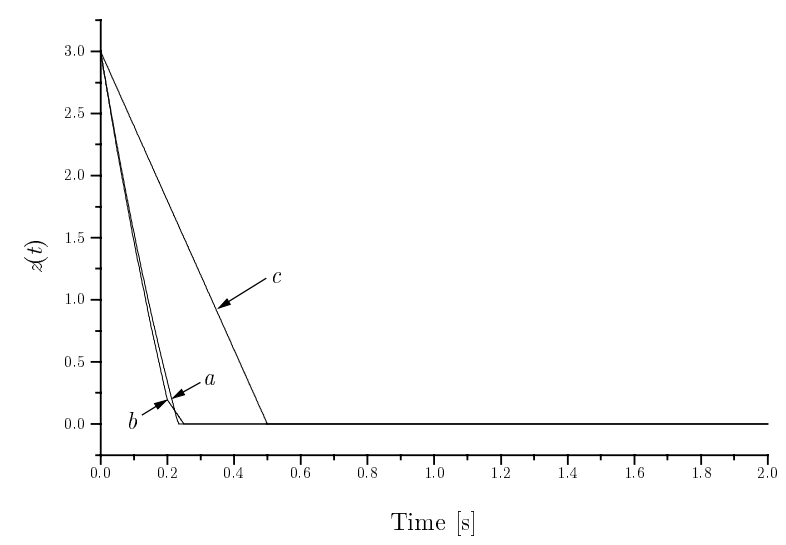

Figure 1. Sliding function $z(t)$ for $T=0.005 \mathrm{~s}$ (curve a) $T=0.05 \mathrm{~s}$ (curve b) $T=0.5 \mathrm{~s}$ (curve c)

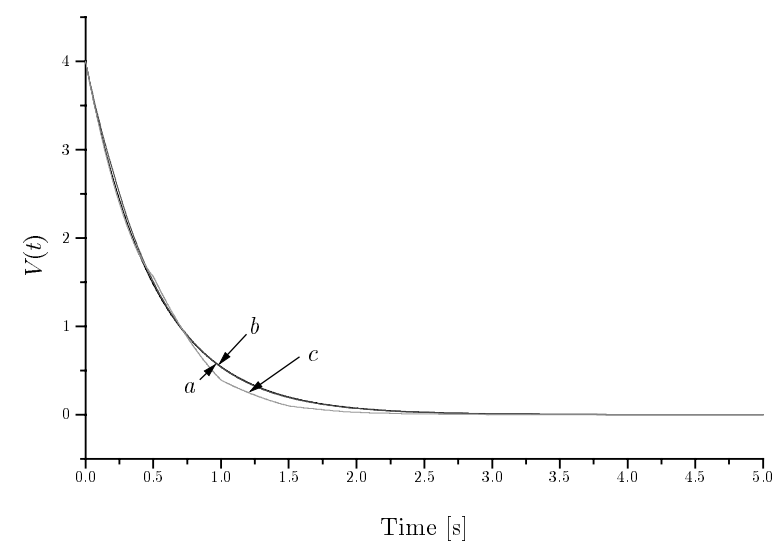

Figure 2. $V(t)$ for $T=0.005 \mathrm{~s}$ (curve a) $T=0.05 \mathrm{~s}$ (curve b) $T=0.5 \mathrm{~s}$ (curve c) 


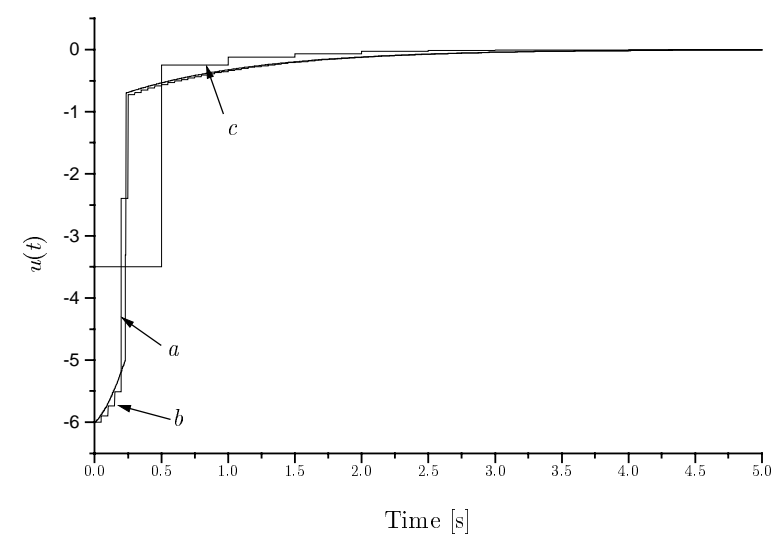

Figure 3. Control signal $u(t)$ for $T=0.005 \mathrm{~s}$ (curve a) $T=0.05 \mathrm{~s}$ (curve b) $T=0.5 \mathrm{~s}$ (curve c)

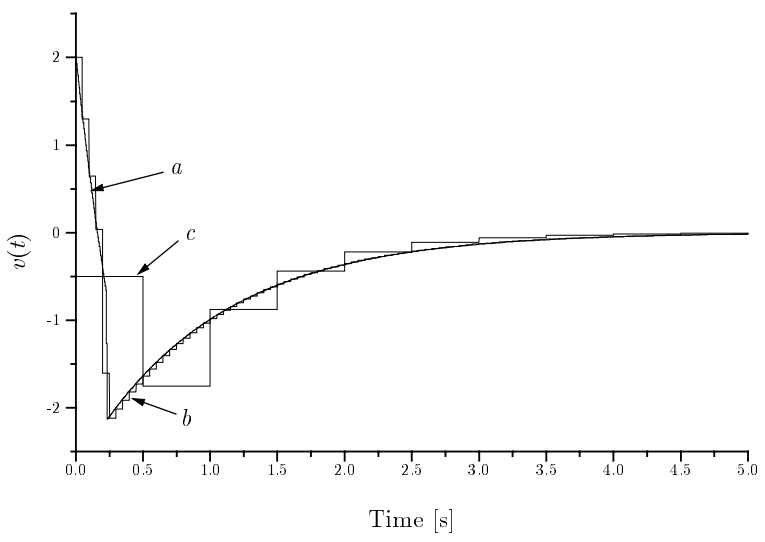

Figure 4. Control signal $v(t)$ for $T=0.005 \mathrm{~s}$ (curve a) $T=0.05 \mathrm{~s}$ (curve b) $T=0.5 \mathrm{~s}$ (curve c)

In Figure 1 it can be seen that the sliding manifold $z=0$ is reached in finite time for all three values of the sampling period $T$. One can see from Figure 2 that the function $V(t)$ asymptotically tends to zero. Therefore, system is stabilized. Figure 3, Figure 4 plot the controls signals $u(t), v(t)$ for the three values of the sampling period. The control signals do not contain high-frequency signal. In other words, the controlled system is chattering free.

Now, the initial condition is $\left[\begin{array}{lll}2 & 2 & 20\end{array}\right]^{\mathrm{T}}$. The parameters $\alpha, \beta$ are chosen as before. For this initial condition it can be shown that the system (25) is stabilized for every $T \in\left(\frac{6}{25}, 2\right)$. Therefore the control law (26) cannot stabilize 
the system (25) for this initial condition. It is chosen that $T=\frac{1}{2}$. It implies that $a \geq 1$. The simulation results are shown in Figure 5 to Figure 8 .

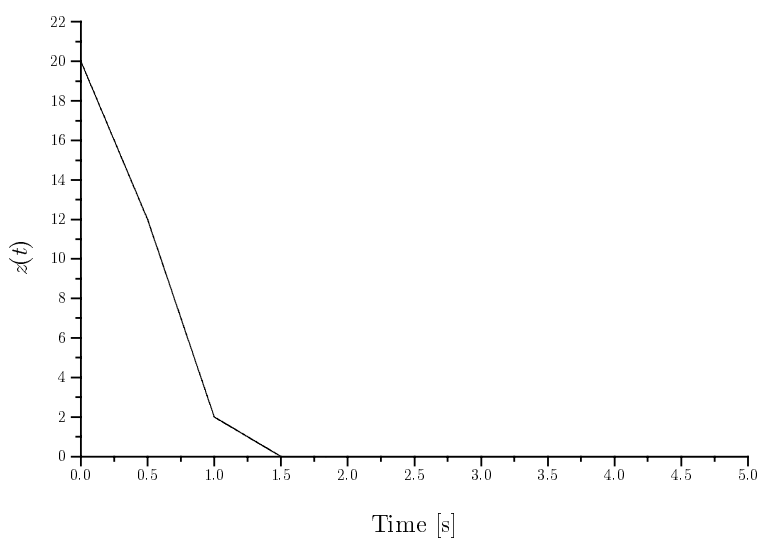

Figure 5. Sliding function $z(t)$

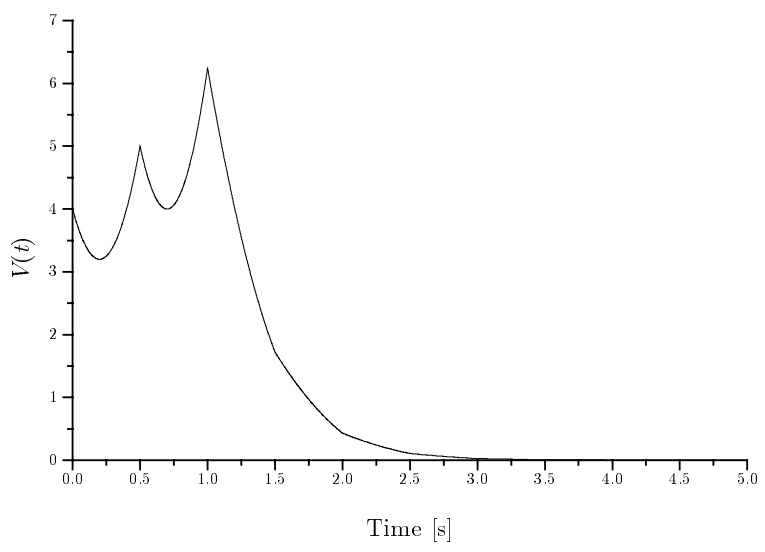

Figure 6. $V(t)$

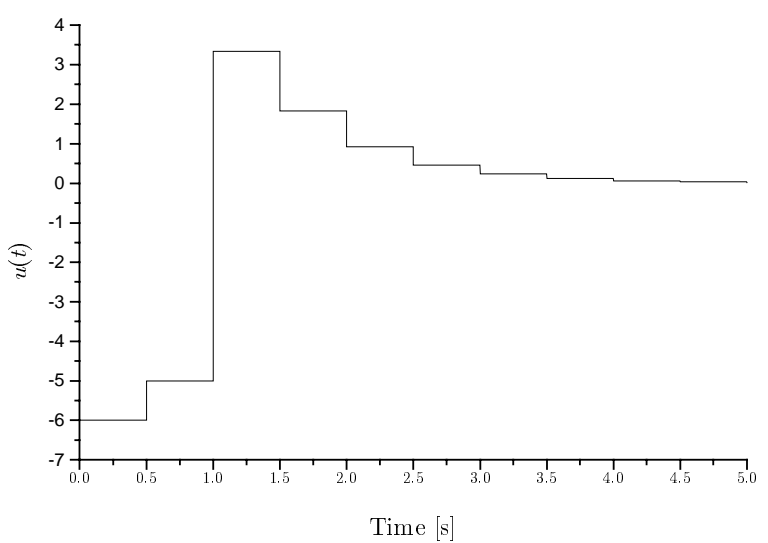

Figure 7. Control signal $u(t)$ 


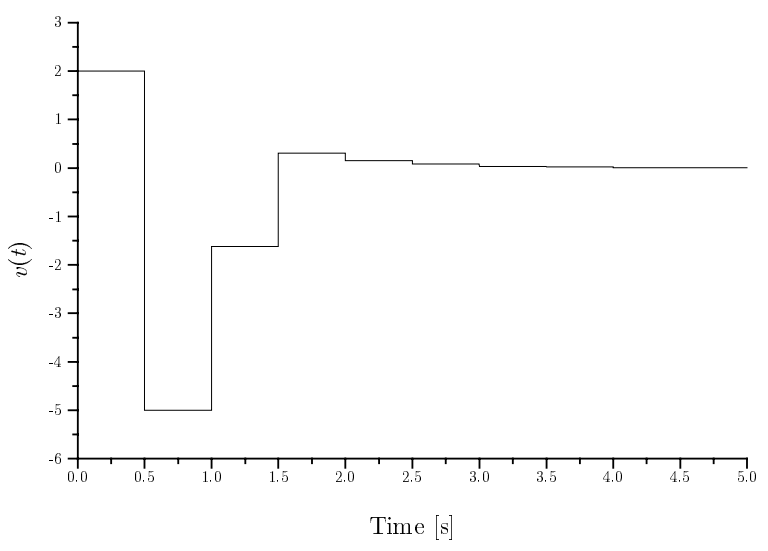

Figure 8. Control signal $v(t)$

Figure 5 clearly shows that the sliding hyperplane is reached in finite number of steps (three steps). Since, $a>1$ then $V_{k}$ is increasing function in two first steps. For $k>3, V_{k}$ is decreasing function since $1-\alpha T=\frac{1}{2}$. Figure 7 and Figure 8 plot the control signals $u(t), v(t)$, respectively.

\section{Conclusion}

In this paper a new method for discretization of CVSVS has been proposed. The approach is based on the linear complementarity theory and obtained control law is such that closed loop system exhibits discrete-time sliding motion in the sense of the definition given by Drakunov and Utkin [6]. Furthermore, the control law is continuous function in respect to variables $x, s, T$ therefore it does not generate disretization chatter. The obtained simulation results given in the section 6 support theory developed in the sections four and five.

The questions remaining to be answered are convergence properties of the signals $\boldsymbol{u}_{k}, \boldsymbol{s}_{k}$. We conjecture that $\boldsymbol{s}_{k}$ converge to $\boldsymbol{s}(t)$ and $\boldsymbol{u}_{k}$ weekly converge to $\boldsymbol{u}(t)$ when $T \rightarrow 0$. This will be one of next direction of our research.

\section{References}

1. Bartolini, G., Ferrara, A., Utkin, V. I., Adaptive Sliding Mode Control in Discrete-time Systems. Automatica 31 (1995) pp. 769-773. 
2. Bloch, A., Drakunov, S., Stabilization and Tracking in the nonholonomic integrator via Sliding Modes. Systems $\&$ Control Letters 29 (1996) pp. 91-99.

3. Chan, C. Y., Servo-systems with Discrete-variable Structure Control. Systems E Control Letters 17 (1991) pp. 321-325.

4. Cottle, R. W., Pang, J. S., Stone, R. E., The Linear Complementarity Problem. (Academic Press, 1992).

5. De Carlo, R., Zak, S. H., Matthews, G. O., Variable Structure Control of Nonlinear Multivariable Systems: A tutorial. Proceeding IEEE 76 (1988) pp. 212-232.

6. Drakunov, S. V., Utkin, V. I., Sliding Mode Control in Dynamic Systems. International Journal of Control 55 (1992) pp. 1029-1037.

7. Drazenovic, B., The Invariance Conditions in Variable Structure Systems. Automatica 5 (1969) pp. 287-295.

8. Furuta, K., Sliding Mode Control of a Discrete System. Systems 86 Control Letters 14 (1990) pp. $145-152$.

9. Gao, W., Hung, J. C., Variable structure Control of Nonlinear Systems: A new Approach. IEEE Transactions on Industrial Electronics 40 (1993) pp.

10. Gao, W., Wang, Y., Homaifa, A., Discrete-Time Variable Structure Control Systems. IEEE Transactions on Industrial Electronics 42 (1995) pp. 117-122.

11. Golo, G., Milosavljević, Č., Robust discrete-time chattering free sliding mode control. Systems and Control Letters 41 (2000) pp. 19-28.

12. Golo, G., Milosavljević, Č., Two-Phase Triangular Wave Oscillator Based on Discrete-time Sliding Mode Controller. Electronic Letters (1997) pp.

13. Hashimoto, H., Ishikawa, Y., Robust Digital Sliding Mode Control Applied to Motion Control Systems. Japan/USA Symposium on Flexible Automation1992) pp. 25-30.

14. Lootsma, Y. J., van der Schaft, A. J., Camlibel, M. K., Uniquness of Solution of Linear Relay Systems. Automatica (1999) pp.

15. Milosavljević, Č., General Conditions for the Existence of Quasi-Sliding Mode on the Switching Hyperplane in Discrete Variable Structure Systems. Avtomatika $i$ Telemekhanika (1985) pp. 36-44.

16. Pfeiffer, F., Glocker, C., Multibody Dynamics with Unilaterl Contacts. (John Wiley \& Sons, 1996).

17. Potts, R. B., Yu, X., Discrete variable Structure System with Pseudo-Sliding Mode. Journal of Australian Mathematical Society 32 (1991) pp. 365-376.

18. Potts, R. B., Yu, X. H., Difference Equation Modeling of a Variable Structure Systems. Computers Mathematical Application 28 (1994) pp. 281-289.

19. Spurgeon, S. K., Hyperplane Design Techniques for Discrete-time Variable Structure. International Journal of Control 55 (1992) pp. 445-456.

20. Utkin, V., Sliding Modes in Control and Optimization. (Springer-Verlag, Berlin, 1992).

21. Utkin, V., Guldner, J., Shi, J., Sliding Mode Control in Electromechanical Systems. (Taylor \& Francis, London, 1999). 
22. Utkin, V. I., Drakunov, S. V., On Discrete-Time Sliding Modes. NOLCOS' 89. (Capri, Italy, 1989) pp. 484-489.

23. Utkin, V. I., Guldner, J., Shi, J., in Systems and Control Series E. Rogers, O. R. J., Eds. (Taylor \& Francis, London EC4P 4EE, 1999).

24. van der Schaft, A. J., Schumacher, H., An Introduction to Hybrid Dynamical Systems. (Springer Verlag, Berlin, 1999).

25. Yu, X., Discrete Variable Structure Control Systems. International Journal of Systems Sciences 24 (1993) pp. 373-386.

26. Yu, X., Potts, R. B., A Class of Discrete variable Structure Systems. 30th Conference on Decision and Control. (Brighton, England, 1991) pp.

27. Yu, X., Potts, R. B., Analysis of Discrete Variable Structure Systems with Pseudo-sliding Modes. International Journal of Systems Sciences 23 (1992) pp. 503-516.

28. Yu, X., Potts, R. B., Computer-controlled Variable-Structure Systems. Journal of Australian Mathematical Society ser. B 34 (1992) pp. 1-17. 